\title{
On the design and simulation of high speed electro-optic modulator based on SOI waveguide MOS dual capacitor structure
}

\author{
Chen Shaowu, Tu Xiaoguang and Yu Jinzhong \\ State Key Laboratory on Integrated Optoelectronics, Institute of Semiconductors, \\ Chinese Academy of Sciences, Beijing 100083 \\ No. Jia35, Qinghua East Rd. Haidian District, P.O. Box 912 \\ Corresponding author: swchen@red.semi.ac.cn Tel: 86-10-82304027
}

Silicon based high speed electro-optic modulator plays important role in silicon OEIC and SOC applications and attracts ever-increasing research interests. In recent years, many novel silicon electro-optic modulators have been proposed and demonstrated, including the silicon ring resonator-based electro-optic modulator proposed by $\mathrm{M}$. Lipson's group, the SOI waveguide electro-optic modulator with MOS capacitor configuration proposed by Liu et al., the SOI waveguide electro-optic modulator based on one-dimensional photonic resonator micro-cavity demonstrated by L.C.Kimerling's group, etc. Generally speaking, the modulators employing p-i-n configuration show high modulation depth but, as opposed to the MOS configuration, a relatively slow operation speed, owing to the slow injection of electrons and holes in forward-bias operation. On the contrary, MOS configuration can achieve high speed, say, $\mathrm{GHz}$ operation, but the modulation depth is rather small, resulting from the poor overlap between the carriers concentration changing region and the waveguide mode. To overcome this problem, M. Lipson's group proposed a micrometer-scale ring resonator-based electro-optic modulator, utilizing the resonating nature of the micro-ring, the transmission at the resonance wavelength is highly sensitive to small index changes, making the modulator can break the slow speed limitation. In this paper, we propose a SOI waveguide modulator with modified MOS dual capacitor structure, by enhancing the overlap between the carriers concentration changing region and the waveguide mode, the modulation depth can be improved greatly, the simulation results support the reasonability of our idea.
The schematic diagram of the proposed structure is shown in Figure 1. A thin film oxide layer, served as the first gate oxide, is formed on the SOI N-type doped top silicon layer $\left(B_{1}\right)$ with thicknes of $t_{B}$ and doping concentration of $1.0 \times 10^{17} \mathrm{~cm}^{-3}$. The core layer (A) is $1.0 \times 10^{17} \mathrm{~cm}^{-3} \mathrm{P}$-type polysilicon deposited on the oxide layer with a thickness of $t_{\mathrm{A}}$. The second gate oxide is formed on the core layer with the same thickness as first oxide layer and covered by a N-type polysilicon upper cladding layer $\mathrm{B}_{2}$. The cross-section of the active region of the modulator is divided into three parts along the vertical direction (y axis) by two gate oxide films, forming the dual capacitor MOS structure. If the device is designed for depletion mode, the upper and lower cladding layers $\mathrm{B}_{1}$ and $\mathrm{B}_{2}$ are P-type doped, or $\mathrm{N}$-type doped for the accumulation mode. Increasing the doping concentration of layer A will result in higher switching speed as well as larger optical absorption loss, so the selected doping concentration here results from a compromise. In order to guarantee the device operating in single-mode and help the electrodes to be formed easily, a double ridge-shaped cross-section structure has been designed. Semi-vector mode BPM is utilized here to analyze the optical field distribution on the cross-section of this structure, the working wavelength of the modulator is set to be $1.55 \mu \mathrm{m}$. Due to the presence of the two gate oxide layers in the active region of the waveguide, the device should be very sensitive to the polarity of incident light. In all the theoretical calculations and simulations presented here, the TE-like fundamental mode is used since TM-like mode induces much lower phase modulation efficiency. Figure 2 shows the optical amplitude distribution in the double ridge-shaped cross-section of the waveguide, indicating one major peak (a) and two minor peaks (b, 
c) formed in the cross section. For MOS structure, the carrier concentration changing region only stays at the very vicinity of the gate oxide layer, resultantly, the large optical field distribution near the gate oxide layer is highly essential to enhance the overlap between optical field and carrier concentration changing region.

At zero bias, no free carrier is accumulated in the vicinity of the gate oxide. A positive bias applied on the core layer (A) will deplete the holes to the anode plane of gate capacitor and collect electrons of same quantity as holes to the cathode plane of gate capacitor at the same time. Owing to the free carriers plasma dispersion effect, variation of the free carriers density overlaped with waveguide mode will induce the change of effective refractive index, thus shifting the lightwave phase. Compared to the structure with single gate capacitor, the dual capacitor structure proposed here increases the overlap between waveguide mode and free carriers concentration changing region, resulting high modulation efficiency and high modulation depth. Intensity modulation can be realized by integrating this structure on the active region of Fabry-Perot cavity, micro-ring resonator or Mach-Zehnder interferometer structure, etc. We simulate the relationship between free carriers density and applied voltage using a commercially available two-dimensional device simulation package ATLAS from SILVACO. Figure. 3 shows the free carriers distribution along the vertical axis under different applied voltage, indicating that the free carriers changing regions only locate near the gate oxide layers and decay rapidly once leaving the gate oxide layers. Strong interaction between optical field and free charge just happens in a $100 \mathrm{~nm}$ thick region. Figure. 4 shows the dependence of phase shift $\Phi$ on the applied gate voltage $V_{g}$ under different length of active region. A phase shift efficiency of $\mathrm{V}_{\pi} \mathrm{L}_{\pi}=3.2(\mathrm{~V} \cdot \mathrm{cm})$ has been achieved.

Small signal response determines the high speed performance of the device. For a normal MOS structure, the switching speed is determined by time constant $\tau=\mathrm{RC}$ only, where $\mathrm{R}$ is controlled by doping concentration of active region, $\mathrm{C}$ is controlled by the thickness of oxide layer and the geometry of waveguide. For accumulation mode, the total capacity is only determined by oxide layer capacity, while for depletion mode, the total capacity is composed of depletion capacity and oxide layer capacity, resulting a smaller capacity and a higher modulation speed. However, device working under depletion mode is limited by threshold voltage $\mathrm{V}_{\mathrm{T}}$, above which the inversion layer will be formed. Under the inversion condition, the changing speed of free carriers concentration in the inversion layer can not keep up with that of applied gate voltage. As a result, only low speed operation (below $100 \mathrm{~Hz}$ ) can be achieved. The dependence of time constant $\tau$ on the doping level $(\mathrm{N})$ and inner-rib width (W) is shown in Fig 5. To obtain high speed performance (exceeding $\mathrm{GHz}$ ), the inner rib width of the waveguide must be smaller than $2.5 \mu \mathrm{m}$ while the doping level keep no less than $1.0 \times 10^{14} \mathrm{~cm}^{-3}$. Fig. 6 shows the modulation speed performance of the device with inner-rib width of $1 \mu \mathrm{m}$, the $10 \%$ to $90 \%$ rise time is $0.1 \mathrm{~ns}$ and the fall time is $0.03 \mathrm{~ns}$, indicating a bandwidth of exceeding $7.5 \mathrm{GHz}$.

In conclusion, a novel Si-based waveguide electro-optic modulator with shunt MOS capacitors is proposed, simulated and analyzed. A positive bias is applied on the special n-i-p-i-n structure to conduct the electro-optic modulation. A phase modulation efficiency of $\mathrm{V}_{\pi} \mathrm{L}_{\pi}=3.2(\mathrm{~V} \cdot \mathrm{cm})$ has been demonstrated for $4 \mu \mathrm{m}$ inner-rib width. For $1 \mu \mathrm{m}$ inner-rib width, the switching speed exceeds $7.5 \mathrm{GHz}$. With the excellent high frequency characteristics, the device is believed to be of great potential for the all silicon-based OEICs applications.

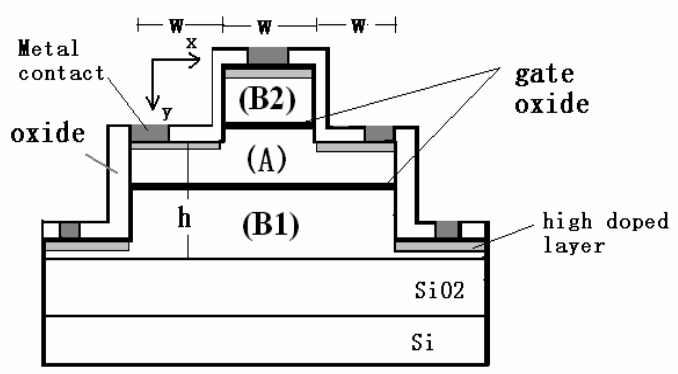

Fig.1. The cross-section of the Si-based waveguide electro-optic MOS modulator 


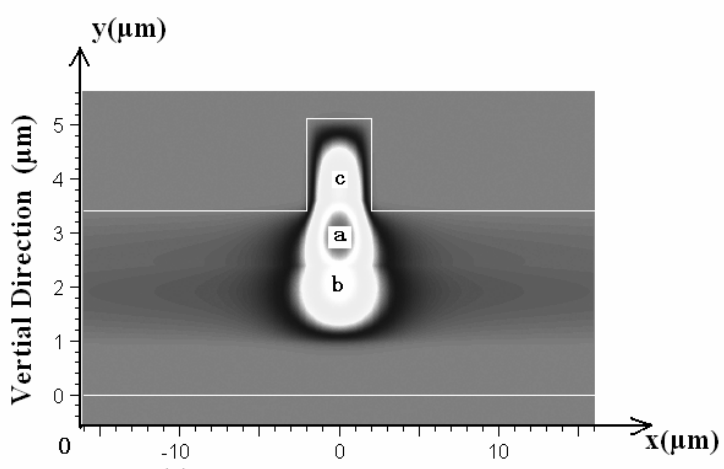

(a) Horizontal Direction $(\mu \mathrm{m})$

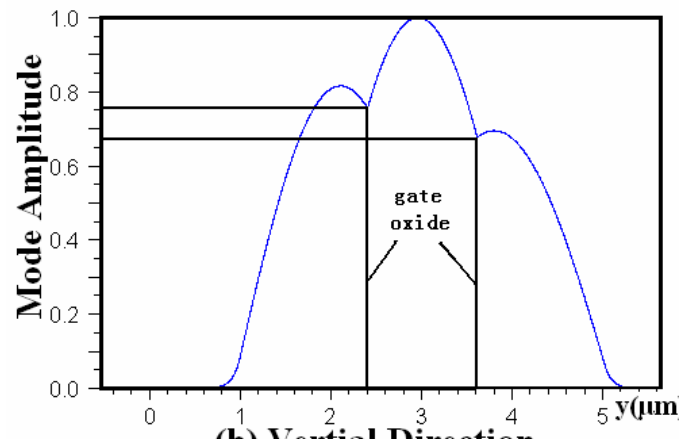

(b) Vertial Direction

Fig.2. (a) TE-like fundamental mode profile on the cross-section of the device (b) Optical field amplitude distribution along the $\mathrm{Y}$ direction at the central line of active region $(x=0)(w=4 u m$, $\mathrm{t}_{\mathrm{A}}=1.2 \mathrm{um}, \mathrm{t}_{\mathrm{B}}=1.4 \mathrm{um}, \mathrm{t}_{\mathrm{ox}}=10 \mathrm{~nm}$ and $\mathrm{h}=2.4 \mathrm{um}$ )
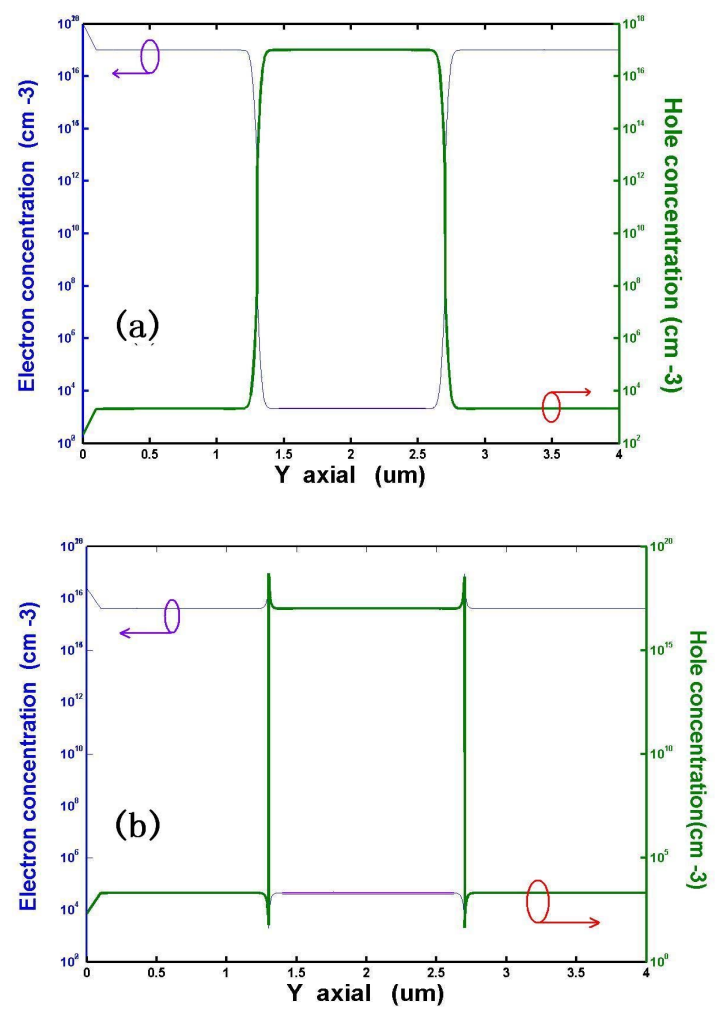

Fig.3. The hole and electron concentration distribution under different applied voltage for the device working in accumulation mode (layer A is p-type doping and layer B is n-type doping by $\left.1 \times 10^{17} \mathrm{~cm}^{-3}\right)$. The parameters are set to be: $t_{A}=1.4 u m, t_{B}=1.3 u m$, $\mathrm{t}_{\mathrm{ox}}=10 \mathrm{~nm}, \mathrm{~W}=4 \mathrm{um}, \mathrm{h}=2.4 \mathrm{um}$, and (a) $\mathrm{V}_{\mathrm{g}}=0 \mathrm{~V}$, ( b) $\mathrm{V}_{\mathrm{g}}=2 \mathrm{~V}$.

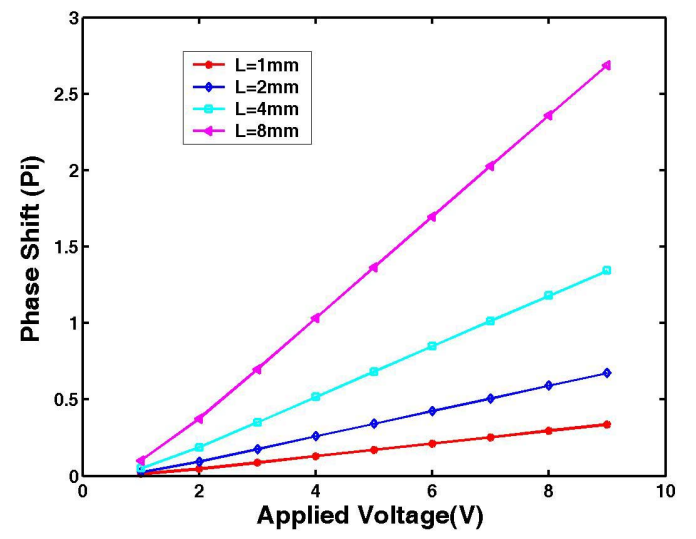

Fig.4. Phase shift vs applied gate voltage for the device working in accumulation mode

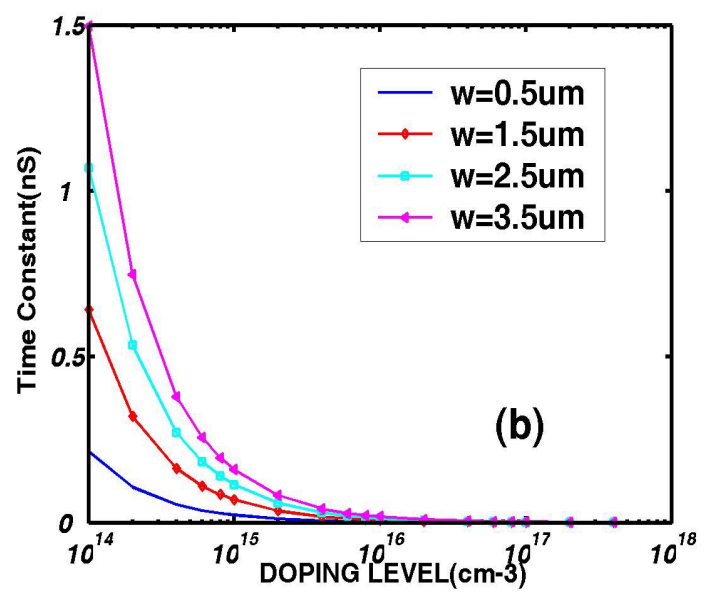

Fig.5. The dependence of time constant on the doping level and inner width (w)

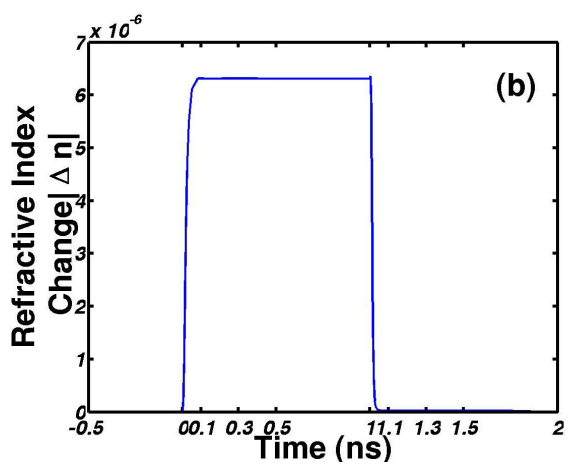

Fig.6. Transient response of the absolute refractive index change to a step driving voltage pulse 